\title{
Beiträge zu einem Verzeichniss der Dipteren Böhmens.
}

\author{
Von Ferdinand Kowarz. \\ V. \\ (Schluss von Seite 208.)
}

Die bei Schiner unter Pipizella und Pipiza vereinigten Gattungen und Arten anderer Autoren unterscheide ich in folgender Weise:

1. Das dritte Fühlerglied schmal und lang . . . . . . 2

- breit und kurz. . . . . . . . . . . . . 3

2. Der Vorderast der ersten Längsader mündet vor der kleinen Querader . . . . . . . . Pipizella Rd.

- hinter der kleinen Querader . . . Heringia Rd.

3. Stirndreieck des ô verhältnissmässig gross und gewölbt; Stirn des ? ohne weisslichen Haarfleck ${ }^{26}$ ) am Augenrande 4

- nicht gross und von gewöhnlicher Bildung; mit einem weisslichen Haarfleck am Augenrande . . . Pipiza Fll.

4. Die hinteren Hüften des O mit dornartigen Fortsätzen; Stirn des $\&$ mit einem deutlichen Quereindruck auf der Mitte

Cnemodon Egg.

- ohne dornartige Fortsätze; - ohne Quereindruck Penium Phil. Heringia: Schwinger gelb; der zweite Hinterleibsring des $\bigcirc$ mit zwei rothen Flecken . . . . . . Alavitarsis $\mathbf{M g}$.

- des or schwarz; - ohne rothe Flecken Zetterstedti Rd.

H. Zetterstedti Rd. - P. leucogona Ztt. (= geniculata $\mathrm{Ztt}$.) wird wahrscheinlich das of von H. Zetterstedti sein; mit P. carbonaria $\mathrm{Mg}$. fällt leucogona - wie Schiner meint - sicher nicht zusammen, weil das $\delta^{c}$ der Zetterstedt'schen Art schwärzliche Schwinger hat; wäre meine Vermuthung richtig, so müsste die in Rede stehende Art den Namen leucogona Ztt. annehmen. - Zetterstedt stellt leucogona allerdings zu denjenigen Pipiza-Arten, welche kürzere Fühler haben; es erübrigt jedoch zu berücksichtigen, dass Z etterstedt diese Art von den mit ihr verglichenen Arten durch „antennis paullo longioribus, angustioribus" unterscheidet und dass analog den Pipizella-Arten das dritte Fühlerglied beim ô kürzer zu sein pflegt als beim ?.

${ }^{26)}$ Bei Pipiza 4-maculata Pz. ist dieser Fleck von braunen Härchen gebildet und daher undeutlich; man erkennt die Art an den vier Flecken, welche sich am zweiten und dritten Hinterleibsringe befinden.

${ }_{n}$ Wiener Entomologische Zeitung" IV. (1. October 1885). Heft 8. 


\section{Tabelle zum Bestimmen der Pipiza-Arten.}

1. Hinterleib mit gelblich durchscheinenden Flecken oder Binden 2 - ohne Flecken oder Binden . . . . . . . . 7

2. Hinterleib am zweiten und dritten Ringe mit je zwei Flecken 4-maculata Pz.

- nur am zweiten Ringe mit zwei Flecken oder einer Binde 3

3. Alle Tarsen ganz gelb, höchstens deren Endglieder gebräunt; der zweite Hinterleibsring des $q$ mit einer gelben Binde festiva Mg.

- schwarz, höchstens die Wurzelglieder ganz oder theilweise gelb; - mit zwei gelben Flecken . . . . . . 4

4. Der Vorderast der ersten Längsader mündet über der kleinen

Querader. . . . . . . . plana Rd. Q

— jenseits der kleinen Querader . . . . . . . 5

5. Gesicht des ठั mit weisslicher Behaarung signata Mg. ठ๊

- schwarzer Behaarung.

6. Hinterleib ganz schwarz behaart $\sigma^{\top}$; Flügel des $O$ unter dem Randmale ohne schwärzlichen Fleck; kleinere schmale Art guttata Mg.

- wenigstens an den Seiten theilweise weisslich behaart $\sigma^{\lambda}$; - mit einem schwärzlichen Fleck ; grössere Art noctiluca L.

7. Flügel unter dem Randmale mit einem schwärzlichen Fleck; Gesicht mit heller Behaarung; grössere Art . . . . 8

- ohne schwärzlichen Fleck; - des $\delta$ mit schwarzer Behaarung; kleinere Art . . . . . stigmatica Ztt. ठ઼

8. Alle Tarsen gelb, höchstens deren Endglieder gebräunt

lugubris $\mathrm{F}$.

- schwarz, höchstens die beiden Wurzelglieder gelb austriaca Mg.

P. 4-maculata Pz. - Dieser Art fehlen bisweilen die Flecken am dritten Hinterleibsringe; man erkennt sie im ठै Geschlechte an dem verhältnissmässig breiten Hinterleib und an der schwarzen Behaarung des ganzen Körpers; dem O fehlen die weisslichen Haarflecken auf der Stirn.

P. signata Mg. - Die Art sieht der P. noctiluca L. überaus ähnlich und unterscheidet sich von dieser durch gerịngere Grösse, durch die weissliche Behaarung des Gesichtes, durch hellere Behaarung am Thorax und durch das heller gefärbte Randmal; auch sind die Flecken am zweiten Hinterleibsring kleiner als bei noctiluca. Von P. geniculata $\mathrm{Mg}$., der sie in der Grösse gleichkommt und mit der sie die helle Behaarung des 
Gesichtes gemeinsam hat, unterscheidet sie sich durch das braune Randmal, das bei geniculata und noctiluca schwarz ist, und durch kleinere Flecken am zweiten Hinterleibsring, insbesondere aber durch die wie bei noctiluca schwarze Behaarung an den Hinterecken der Hinterleibsringe und am Hypopygium; bei geniculata ist diese Behaarung längs den Hinterleibsseiten und am Hypopygium durchaus weisslich.

P. guttata Mg. - Schiner bezeichnet die Vordertarsen dieser Art als "ganz gelb", während Meigen sie blos "gelbhaarig" nennt.

P. lugubris F. - Ich bin der Meinung, dass die Art, welche Schiner als lugubris beschrieben hat, der dunklen Tarsen wegen zu austriaca Mg. gehört und dass austriaca Schin. die richtige $P$. lugubris $\mathrm{F}$. ap. $\mathrm{Mg}$. ist; denn $\mathrm{M}$ eige $\mathrm{n}$ nennt ausdrücklich bei seiner lugubris die "Füsse rostgelb“ und bei austriaca die "Füsse rostgelb behaart".

Tabelle zum Bestimmen der Cnemodon-Arten.

1. Metatarsus der Vorderbeine breiter als das folgende Tarsenglied; die Beule auf der Vorderseite der Mittelschienen sehr auffallend . . . . . . . latitarsis Egg. $\sigma^{\top}$

- nicht breiter als das folgende Tarsenglied; - weniger auffallend .

2. Schwinger schwarz

morionellus Ztt. ठَ

- gelb.

3. Behaarung des Thoraxrückens vorherrschend schwarz

\section{— weisslich .. . . . . . brevidens Egg. ठ}

Cn. latitarsis Egg. variirt wie die anderen CnemodonArten in der Färbung der Vordertarsen; Pipiza vitripennis $\mathrm{Mg}$. und $P$. anthracina $\mathrm{Ztt}$. dürften mit $C n$. latitarsis identisch sein.

Cn. morionellus Ztt. ist im $\sigma$ Geschlechte durch die schwarzen Schwinger und in beiden Geschlechtern durch das relativ grössere, auf der Unterseite gelb gefärbte dritte Fühlerglied von fulvimanus Ztt. zu unterscheiden. Es ist nicht unmöglieh, dass auch ruficornis $\mathrm{Mg}$. \& $\mathrm{zu}$ morionellus gehört.

Penium carbonarium $\mathrm{Mg}$. - Ich bin über die richtige Deutung dieser Art nicht völlig sicher und lasse daher deren ergänzende Beschreibung hier folgen:

ธิ Kopf, Thorax und Hinterleib glänzend schwarz und schwarz behaart; Stirn und Gesicht gewölbt, mit schwach- 
blauem Schimmer; Fühler braun, das dritte Glied kurz und verkehrt eiförmig; Schildchen nicht gerandet; Flügel bräunlich, an der Wurzelhälfte lichter; Randmal bräunlichgelb; der Vorderast der ersten Längsader mündet etwas jenseits der kleinen Querader, die Spitzenquerader hinter ihrer Mitte geschwungen, d. h. die Schwingungsstelle dieser Querader ist der vierten Längsader näher als der dritten, an der Verbindungsstelle mit der schwach nach vorn gebogenen dritten Längsader einen ziemlich spitzen, inneren Winkel bildend; Schüppchen weisslich, mit schwärzlichem Rande; Schwinger mit rostgelbem Knopfe. Beine einfach, schwarz und schwarz behaart, das filzartige Toment an den Schienen und Tarsen rostgelb schimmernd; alle Kniee und der Metatarsus der Mittelbeine an der Basis rostgelb; der Metatarsus der Hinterbeine kaum verdickt. Hinterleib ziemlich schlank, matt und nur an den Seiten so wie das Hypopygium glänzend; die Behaarung: des Hinterleibs an den Seiten desselben heller schimmernd.

† Kopf, Thorax und Hinterleib bläulich schwarz, fein punktirt, mässig glänzend, mit kurzer, weisslicher Behaarung, welche nur am Scheitel und vor den Ocellen länger und schwarz ist; Stirn fast gleich breit, ohne deutlichen Eindruck auf der Mitte, am oberen Theile - soweit dieser von der schwarzen Behaarung bedeckt ist - etwas über die Stirnfläche erhoben; Fühler etwas länger und breiter als beim $\delta$, rostgelb, das dritte Glied auf der Oberseite und an der Spitze gebräunt. Flügel etwas breiter, die Spitzenquerader etwas steiler als bei dem $\widetilde{\sigma}$; Deckschüppchen blassgelb. Die gelbe Färbung der Beine besonders an den vorderen Schienen ausgebreiteter, der Metatarsus der Mittelbeine und die Basis des folgenden Gliedes ebenso gefärbt.

Grösse $6.5 \mathrm{~mm}$.

Die Detailzeichnungen, welche Philippi l. c. von seinen beiden Penium-Arten entwirft, passen im Allgemeinen auf $P$. carbonarium; die vena spuria ist bei ihm vorhanden. Ich glaube die Gattung Penium richtig gedeutet $\mathrm{zu}$ haben, wenn ich auf die Zeichnungen Philippi's mehr Gewicht lege, als auf seine bezüglich der "cellula submarginalis pediformis" mit ihnen im Widerspruche stehende Gattungsdiagnose. 


\section{$2 \mathrm{BHL}$ Biodiversity Heritage Library}

Kowarz, Ferdinand. 1885. "Beiträge zu einem Verzeichnisse der Dipteren Böhmens. V. Fortsetzung." Wiener entomologische Zeitung 4, 241-244. https://doi.org/10.5962/bhl.part.20134.

View This Item Online: https://www.biodiversitylibrary.org/item/44101

DOI: https://doi.org/10.5962/bhl.part.20134

Permalink: https://www.biodiversitylibrary.org/partpdf/20134

\section{Holding Institution}

Smithsonian Libraries

\section{Sponsored by}

Smithsonian

\section{Copyright \& Reuse}

Copyright Status: NOT_IN_COPYRIGHT

This document was created from content at the Biodiversity Heritage Library, the world's largest open access digital library for biodiversity literature and archives. Visit BHL at https://www.biodiversitylibrary.org. 